See discussions, stats, and author profiles for this publication at: https://www.researchgate.net/publication/341647767

\title{
Positive Dynamical Networks in Neuronal Regulation: How Tunable Variability Coexists with Robustness
}

Article · May 2020

DOI: 10.1109/LCSYS.2020.2997214

CITATIONS

READS

0

34

3 authors, including:

Alessio Franci

Universidad Nacional Autónoma de México

65 PUBLICATIONS 581 CITATIONS

SEE PROFILE

Some of the authors of this publication are also working on these related projects:

ERC Switchlets View project 


\title{
Positive dynamical networks in neuronal regulation: how tunable variability coexists with robustness.
}

\author{
Alessio Franci, Timothy O’Leary and Jorge Golowasch
}

\begin{abstract}
Neuronal systems exhibit highly stable and tunable behaviors in spite of huge variability at the molecular component level and in spite of persistent physiological and pathological perturbations. How is this robust flexibility achieved? Homeostatic integral control has been shown to be key in reconciling variability with stability, but the explanatory model used lacks basic robustness properties to perturbations. We suggest that positive molecular regulatory networks may play a major role in reconciling stability, variability and robustness. The idea we propose is that integral control happens along the dominant direction of the network. This slow direction generates a strongly attractive, and thus robust, subspace along which almost perfect homeostatic regulation can be achieved. Fluctuations of relevant molecular variables along this positive dominant subspace explain how big, positively-correlated variations of biophysical parameters (as measured in experiments) are compatible with robust regulation, thus explaining flexibility. Because of robustness, the properties of the positive network can be subject to slower tuning processes (like the circadian rhythm), which provides a biologically plausible basis for tunable variability to be compatible with robust regulation. The relevance of the proposed regulation model for control-theoretical approaches to neurological diseases is also discussed.
\end{abstract}

\section{INTRODUCTION}

Neurons must continuously adapt their electrical activity to shape information transmission through the central nervous system in a task and state-dependent fashion. For instance, during sleep, the electrical activity of neurons in the sensory thalamus and all across the cortex exhibits a specific type of pattern, called bursting, that blocks incoming sensory information and allows for crucial sleep-related plasticity processes [13]. During arousal, the electrical activity is sharply different and characterized by a slow tonic-spiking pattern that allows faithful and almost linear encoding of sensory inputs. The switch between the two modes can and should happen reliably, for instance, during transitions between wakefulness and sleep. At the same time, the biophysical parameters determining neuronal activity exhibit huge degrees of variability even between cells of the same type [11], [8] and, as any other biological system, neurons must maintain a suitable

This work was supported in part by UNAM-DGAPA-PAPIIT grant IN102420 and by CONACyT grant A1-S-10610 and in part by US National Science Foundation (NSF) DMS1715808.

Alessio Franci is with the Department of Mathematics, National Autonomous University of Mexico, Ciudad Universitaria, 04510, Mexico City, Mexico afrancieciencias.unam.mx

Timothy O'Leary is with Department of Engineering, University of Cambridge, Cambridge CB2 1PZ, UK tso24 e cam. ac.uk

Jorge Golowasch is with Federated Department of Biological Sciences New Jersey Institute of Technology \& Rutgers Univ-Newark, Newark, NJ 07103, USA golowasch@njit.edu

978-1-5386-5541-2/18/\$31.00 @ 2018 IEEE homeostatic activity level to avoid runaway excitation, lack of activity, or other neuronal dysfunctions [18]. How is robust homeostatic regulation achieved in spite of ongoing modulation and parameter variability remains a debated topic [12], [9].

The model derived in [17] shows that a biologically plausible integral control scheme (described in some details in Section II) can reconcile homeostatic regulations with variability and modulation. It also provides a mechanism to explain the key experimental observation that biophysical parameter variations in a same neuronal type are big but positively correlated [19]. Despite its success in explaining key features of neuronal regulation, the model proposed in [17] lacks basic robustness properties, which clash with recent findings in molecular and circadian biology. The molecular basis of the proposed control scheme involves the regulation of gene transcription rates. Transcription rates are highly noisy for a large part of the genome and robust expression of transcription products (including mRNA) is mainly achieved at the posttrascriptional level by molecular regulatory networks [14], [2]. The integral model proposed in [17] leads to unbounded molecular and biophysical parameters as soon as noisy transcription is considered, as proved in this paper. Secondly, it has recently been shown [21] that the positive linear set along which biophysical parameter vary can undergo dramatic changes during the circadian period. Because of its sensitivity to initial conditions, the neuronal regulator model in [17] cannot reproduce these results.

Motivated by these observations, we propose to extend the model in [17] with a generic positive $e^{1}$ molecular regulatory network, modeling post-transcription regulation of mRNA expression. The main idea we propose is that the network dominant (slow) direction provides a tunable space along which regulation is possible, robustly to all kind of disturbances and to initial conditions.

The paper is organized as follows. In Section II we review the model in [17] from a control theoretical perspective and use transfer function analysis to highlight its key features and limitations. In Section III we introduce the revised extended model and use singular perturbations and transfer function analysis to analyze its robust behavior from both a mathematical and a biological perspective. In Section IV we reproduce the results of numerical simulations on a detailed computational model to further illustrate the biological plausibility of the proposed control scheme. Finally, we discuss the relevance of the proposed ideas for control theoretical approaches to neurological diseases in Section V.

\footnotetext{
${ }^{1}$ Positivity is intended here in the sense of cooperative or mostly cooperative interactions between the nodes of the network, which ensures the existence of a positive dominant eigenvector. See Section III for details.
} 


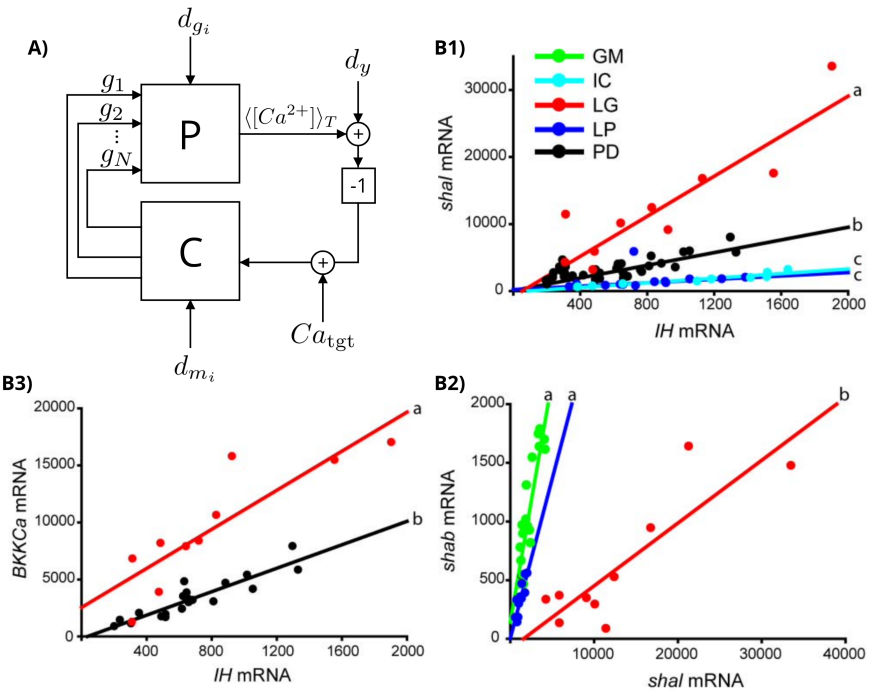

Fig. 1. Molecular regulation of ion channel expression. A. Block diagram representation of neuronal regulation via a molecular feedback loop. $P$ is the neuronal plant, which maps maximal conductances $g_{1}, \ldots, g_{N}$ into electrical activity. On the molecular timescale, electrical activity is reported by the output $\left\langle\left[\mathrm{Ca}^{2+}\right]\right\rangle_{T}$, which is regulated by a molecular controller $C$. $d_{g_{i}}, d_{y}, d_{m_{i}}$, are the plant, output, and control disturbances, respectively. B. Experimentally measured correlations between channel mRNA levels in five distinct neuron types of the crab stomatogastric nervous system (color coded, each point is a different cell). Each neuron type is characterized by a distinct linear subspace in the space of ion channel expression. Neurons of the same type (and activity) can possess very different biophysical parameters as long as correlations between them are preserved. B is adapted from [19, Figure 5]

\section{A SIMPLE NEURONAL REGULATOR AND ITS LIMITATIONS}

\section{A. A simple model of neuronal activity}

From a regulation viewpoint, the electrical activity of a neuron can be modeled as a plant $P$ that maps maximal conductances $g_{i}$ to the intracellular calcium concentration (Figure 1A). Maximal conductances are biophysical parameters that determine the neuron electrical behavior by fixing the strength of ionic currents flowing through the neuron membrane. They are the key parameters in determining the neuronal excitable behavior [8] and are subject both to molecular and neuromodulatory regulation [12]. Considering intracellular calcium as the output of a neuron is natural because: $i$ ) it is a good reporter of the amount of ionic current flowing through the membrane; and ii) it couples the neuronal electrical activity to the cell molecular machinery by activating a variety of second messenger pathways and transcription regulators [5].

The resulting plant I/O relationships are in general dynamical and highly nonlinear, because of the complicated electrical neuronal dynamics (spiking, bursting, etc.) [8]. However, because the electrical dynamics are much faster than the cell molecular dynamics they are coupled $\mathrm{to}^{2}$, one can make two simplifications when studying neuronal regulation mediated by molecular pathways. First, the electrical dynamics can be treated as infinitely fast, i.e., instantaneous. Second, electrical dynamics can be averaged over long time windows to get rid

\footnotetext{
${ }^{2}$ The time scales of neuronal electrical dynamics are in the order of a fraction of millisecond to a few second. Molecular dynamics evolve on timescales of minutes to hours.
}

of intrinsic fast electrical oscillations like spikes and bursts. In summary, from a regulation viewpoint, a neuron can be modeled as a static map $\left\langle\left[C a^{2+}\right]\right\rangle_{T}(t)=C(g(t))$, where $\left\langle\left[\mathrm{Ca}^{2+}\right]\right\rangle_{T}$ is the average intracellular calcium concentration over a sufficiently long (minutes, for instance) time interval $[t-T, t]$. Linearizing around a desired set point, we can approximate this map as the linear relationship $\left\langle\left[\mathrm{Ca}^{2+}\right]\right\rangle_{T}=$ $c^{T} g$, where $g=\left(g_{1}, \ldots, g_{N}\right)$ is the vector of maximal conductances, $c=\left(c_{1}, \ldots, c_{N}\right)$, and $c_{i}>0$ (resp. $\left.c_{i}<0\right)$ signifies that the ionic conductance $i$ tends to increase (resp. decrease) $\left\langle\left[\mathrm{Ca}^{2+}\right]\right\rangle_{T}$.

\section{B. A simple model of neuronal regulation}

Each ionic current type flows through a specific type of ion channel carrying specific types of ions across the cell membrane. Ion channels are proteins embedded in the cell membrane. The maximal conductance of a given ionic current is proportional to the number of membrane ion channels carrying that current. How many ion channels of a given type are available in the membrane, and thus how large is the resulting ionic current maximal conductance, is largely regulated by the rates of expression of the genes coding for each ion channel protein. In turn, gene expression rates are dependent on intracellular calcium levels [5]. A major biological hypothesis in [17] is that gene expression rates are regulated in such a way that $\left\langle\left[\mathrm{Ca}^{2+}\right]\right\rangle_{T}$ is maintained around a desired set point $C a_{\mathrm{tgt}}$, coded in the cell's physiology (see [17, Figure 1A]). The main modeling proposal in [17] is then that ion channel expression is regulated by an integral controller $C$ that achieves perfect regulation of $\left\langle\left[\mathrm{Ca}^{2+}\right]\right\rangle_{T}$ (see Figure 1A and [17, Figure 1B]). The resulting closedloop integral controller equations can be simplified as

$$
\begin{aligned}
\tau_{i} \dot{m}_{g_{i}} & =C a_{\mathrm{tgt}}-\left\langle\left[C a^{2+}\right]\right\rangle_{T}+d_{y}+d_{m_{i}} \\
& =C a_{\mathrm{tgt}}-c^{T} g+d_{y}+d_{m_{i}} \\
\tau_{g} \dot{g}_{i} & =-g_{i}+m_{g_{i}}+d_{g_{i}} .
\end{aligned}
$$

where $m_{g_{i}}$ is the concentration of a generic channel protein precursor (including messenger RNA (mRNA)) associated to ionic current of type $i, g_{i}$ is the associated maximal conductance, and $d_{y}, d_{m_{i}}, d_{g_{i}}$ are disturbances. With a small biological abuse of notation, we will refer to the $m_{g_{i}}$ simply as the mRNA concentrations or expression levels associated to the maximal conductances $g_{i}$. There can be an arbitrary large number $(N \in \mathbb{N})$ of ionic current types, so $i=1, \ldots, N$. The positive constants $\tau_{i}, \tau_{g}$ fix the time scales of mRNA transcription from genes and translation to proteins, respectively. In model (1), we consider output disturbances $d_{y}$ and two types of state disturbances: $d_{g_{i}}$, i.e., maximal conductance disturbances, and $d_{m_{i}}$, i.e., mRNA disturbances.

Let $G_{z \rightarrow w}(s)$ denote the transfer function from $z$ to $w$ associated to model (1), where $z$ and $w$ are two signals. Then we have the following proposition, whose proof follows by straightforward computations and is omitted.

Proposition 1. Let $q=\sum_{i=1}^{N} c_{i} / \tau_{i}$ and $T(s)=s\left(\tau_{g} s+1\right)$.

- $G_{r \rightarrow e}(s)=\frac{T(s)}{q+T(s)}$; 
- $G_{d_{g_{i}} \rightarrow e}(s)=-c_{i} \frac{s}{q+T(s)}$;

- $G_{d_{y} \rightarrow e}(s)=-\frac{q}{q+T(s)}$;

- $G_{d_{m_{i}} \rightarrow e}(s)=-\frac{c_{i} / \tau_{i}}{q+T(s)}$.

Observing that $T(0)=0$, it follows that if $q>0$ and $d_{y}=d_{m_{i}}=0$ the control scheme proposed in [17] achieves perfect regulation of $\left\langle\left[\mathrm{Ca}^{2+}\right]\right\rangle_{T}$ that is robust to constant maximal conductance disturbances. It also achieves robust compensation of all disturbance types, in the sense that all transfer functions from disturbances to output errors have only poles with negative real parts. The condition $q>0$ constraints the values of the $\tau_{i}$ 's to be smaller, i.e., mRNA expression faster, for conductances that tend to increase $\left\langle\left[\mathrm{Ca}^{2+}\right]\right\rangle_{T}$. This observation suggests an experimentally testable connection between homeostatic regulation and channel expression rate.

In the absence of maximal conductance and mRNA disturbances, the closed-loop neuronal regulation model (1) also provides a mechanism to "develop" a neuronal type. Various experimental observations [19], [22] revealed that a given neuronal type is defined by linear relationships between its maximal conductances, rather than by a single point in the maximal conductance space, as illustrated in Figure 1B (adapted from [19, Figure 5]). Formally, a neuronal type corresponds to a bounded subset of the linear subspace $\left\{g_{i}=\alpha_{i j} g_{j}\right\}$. We say that two conductances $i, j$ are positively correlated in a given neuronal type if $\alpha_{i j}>0$, negatively correlated if $\alpha_{i j}<0$, uncorrelated if $\alpha_{i j}=0$. The insight from localized nonlinear sensitivity analysis [3] is that conductance correlations correspond to a balance of distinct ionic currents [6]. Experimentally, $\alpha_{i j} \geq 0$ for all $i=1, \ldots, N$ and all $j=1, \ldots, k$, i.e., all conductance pairs are either positively correlated or uncorrelated in biological systems. Starting at time $t=0$ from an non-differentiated neuronal cell, i.e., one with $m_{g_{i}}(0) \approx g_{i}(0) \approx 0$, the homeostatic controller (1) with $d_{g_{i}}=d_{m_{i}}=d_{y}=0$ develops the neuronal type associated to the positive linear correlations

$$
\frac{g_{i}}{g_{j}} \approx \frac{\tau_{j}}{\tau_{i}} .
$$

Indeed, in the absence of disturbances, $m_{g_{i}}(t)=$ $\frac{1}{\tau_{i}} \int_{0}^{t} e(\tau) d \tau+m_{g_{i}}(0)$. Thus, once the integral controller has converged, (2) is satisfied provided initial conditions are sufficiently small.

\section{Limitations of the simple regulation model}

The first, crucial limitation of model (1) is that, although the neuronal output $\left\langle\left[\mathrm{Ca}^{2+}\right]\right\rangle_{T}$ can be robustly regulated, the presence of disturbances leads to unbounded molecular and biophysical parameters. The simple computations needed to prove the following proposition are omitted.

Proposition 2. Let $q=\sum_{i=1}^{N} c_{i} / \tau_{i}$ and $T(s)=s\left(\tau_{g} s+1\right)$.

- $G_{d_{m_{i}} \rightarrow g_{i}}(s)=\frac{1}{T(s)}\left(1-\frac{c_{i} / \tau_{i}}{q+T(s)}\right)$;

- $G_{d_{m_{j}} \rightarrow g_{i}}(s)=-\frac{1}{T(s)} \frac{c_{j} / \tau_{j}}{q+T(s)}$.

Because $T(s)=0$, any mRNA disturbance with non-zero zero-frequency component (for instance, any colored noise)

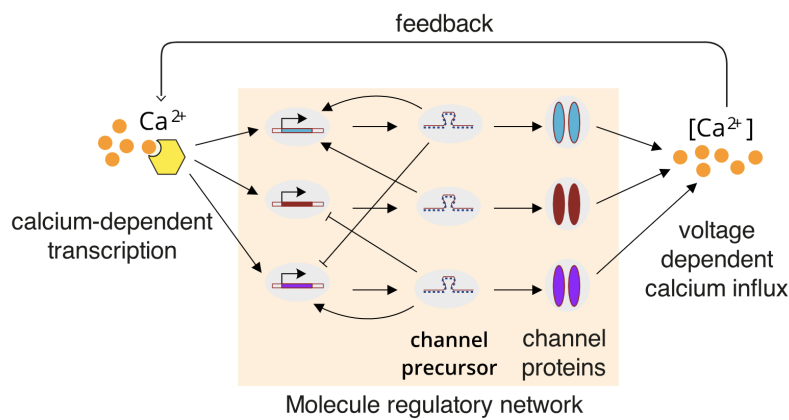

Fig. 2. Post-transcriptional neuronal regulation. This homeostatic controller is similar to the one proposed in [17] but includes a network of molecular interactions that controls ion channel expression, rather than simple gene-tochannel regulation.

generically leads to unbounded maximal conductances, which is meaningless biologically.

Remark 1. Observe that if $d_{m_{1}}=\cdots=d_{m_{N}}=d_{m}$, then $G_{d_{m} \rightarrow g_{i}}(s)=\sum_{j=1}^{N} G_{d_{m_{j}} \rightarrow g_{i}}(s)=\frac{1}{q+T(s)}$ have only poles with negative real part and thus the controller is robust (i.e., it does not lead to unbounded states) to this specific type of disturbances. In [17] a discussion of this fact is provided in terms of homogeneous versus heterogeneous calcium targets for the various mRNA expression rates.

The second limitation of model (1) is its sensitivity to initial conditions. Indeed, the model develops conductance correlations as defined by (2) only for very small initial conditions. Starting from generic initial conditions the model will let conductances converge to a completely different set as a function of the specific initial condition values. In other words, the linear correlation set defined by (2) is not attractive in model (2). This clashes with the recent observations in [21], which show that, along the circadian cycle, conductances robustly and periodically move between different correlation sets, or get uncorrelated at a given time of day and converge back to a correlation set at another time of the day. To reproduce these experimental observations, the linear correlation set should be attractive.

\section{A SIMPLE AND ROBUST NEURONAL REGULATOR}

A key biological feature that is missing in the integral control model developed in [17] is that once transcribed, the channel mRNAs $m_{g_{i}}$ enter complicated post-transcriptional regulatory networks that can drastically change the amount of translated channel proteins as compared to the amount of transcribed mRNA [14]. The results in [14] reveal that a post-transcriptional process coherently orchestrates mRNA expression and that mRNA transcription is highly noisy for the larger part of the genome. We thus introduce a generic post-trascriptional molecular regulatory network between the 
transcribed channel precursors $m_{g_{i}}$ as follows ${ }^{3}$

$$
\begin{aligned}
& \dot{m}_{g_{i}}=\left(C a_{\mathrm{tgt}}-\left\langle\left[C a^{2+}\right]\right\rangle_{T}\right)+\sum_{j=1}^{N} A_{i j} m_{j}+d_{y}+d_{m_{i}} \\
& \tau_{g} \dot{g}_{i}=-g_{i}+m_{g_{i}}+d_{g_{i}} .
\end{aligned}
$$

See Figure 2 for a graphical representation of the extended controller.

Assumption 1. The network dynamics (3) are stable (i.e., A has all eigenvalues with non-positive real part). Moreover, A possesses a dominant positive eigenvector. That is, A possesses a real (dominant) eigenvalue $\lambda_{1}$ such that: $i$ ) the other eigenvalues $\lambda_{2}, \ldots, \lambda_{N}$ of A satisfy $\operatorname{Re}\left(\lambda_{i}\right)<\lambda_{1}$; ii) $\lambda_{1}$ has algebraic multiplicity one; iii) the associated eigenvector $v_{1}$ is positive.

The key idea we propose is that the subspace generated by the positive dominant eigenvector of the network determines an attractive, robust, slow direction along which conductances are positively correlated and along which (almost) perfect integral control is possible. The following remark clarifies the connection between Assumption 1 and positive dynamics.

Remark 2. Assumption 1 is satisfied in the following important cases:

1) the linear dynamics (3) are positive, in the sense that positive inputs leave the positive orthant invariant, that is, if and only if $A$ is Metzler [1, Lemma 8.1];

2) there exists $\delta \in \mathbb{R}$ such that $A+\delta I$ is eventually positive, that is, there exists $n \in \mathbb{N}$ such that $(A+\delta I)^{n}$ is positive. Eventual positivity is equivalent to eventual exponential positivity [7, Theorem 5].

From a biological viewpoint, both case 1) and 2) occur when most of the molecular interactions underlying the neuronal homeostatic regulator are cooperative. In case 1), $A$ possesses a positive dominant eigenvector because all Metzler matrices do. To prove that the same is true in case 2), we invoke [16, Theorem 2.2], which implies that $A+\delta I$ possesses a positive dominant eigenvector and so does $A$.

Let $v_{2}, \ldots, v_{N}$ be a basis for the generalized eigenspace associated to the eigenvalues $\lambda_{2}, \ldots, \lambda_{N}, \operatorname{Re}\left(\lambda_{i}\right)<\lambda_{1} \leq 0$. Then, there exists a change of coordinate $U$, with columns $U_{\cdot i}=v_{i}$, such that $\left[m_{g_{i}}\right]_{i=1}^{N}=U \tilde{m}$ and $\tilde{m}$ is ruled by a block-diagonal dynamics ${ }^{4}$

$$
\begin{gathered}
\dot{\tilde{m}}_{1}=\lambda_{1} \tilde{m}_{1}+b_{1}\left(e+d_{y}\right)+\left\langle U_{1}^{-1},\left[d_{m_{i}}\right]_{i=1}^{N}\right\rangle \\
{\left[\dot{\tilde{m}}_{i}\right]_{i=2}^{N}=\tilde{A}\left[\tilde{m}_{i}\right]_{i=2}^{N}+\left[b_{i}\right]_{i=2}^{N}\left(e+d_{y}\right)+} \\
+\left\langle U_{i \cdot}^{-1},\left[d_{m_{j}}\right]_{j=1}^{N}\right\rangle_{i=2}^{N}, \\
e=r-y=C a_{\mathrm{tgt}}-\left\langle\left[C a^{2+}\right]\right\rangle_{T},
\end{gathered}
$$

with $b_{i}=\sum_{j=1}^{N} U_{i j}^{-1}$ and $\tilde{A}$ and $(N-1) \times(N-1)$-dimensional matrix with spectrum $\lambda_{2}, \ldots, \lambda_{N}$. In particular, $\tilde{A}$ and all transfer functions associated to (4) are Hurwitz.

\footnotetext{
${ }^{3}$ For simplicity and to reduce the number of parameters, all the $\tau_{i}$ 's were set to one. The analysis below can easily be generalized to the case of different $\tau_{i}$ 's by suitably scaling the rows of the matrix $A$.

${ }^{4}\langle\cdot, \cdot\rangle$ denotes the Euclidean scalar product.
}

The behavior of the closed-loop dynamics (3) can be understood in the limit in which the fast eigenmodes associated to the eigenvalues $\lambda_{2}, \ldots, \lambda_{N}$ are much faster than the slow mode associated to dominant eigenvalue $\lambda_{1}$. Let

$$
\varepsilon=\frac{1}{\min _{j=2, \ldots, N}\left|\operatorname{Re}\left(\lambda_{j}\right)\right|} .
$$

Theorem 1. The slow dynamics associated to the singular limit $\varepsilon \rightarrow 0$ of model (3) is

$$
\begin{aligned}
\tau_{g} \dot{g}_{i} & =-g_{i}+\left[v_{1}\right]_{i} \tilde{m}_{1}+d_{g_{i}} \\
\dot{\tilde{m}}_{1} & =\lambda_{1} \tilde{m}_{1}+b_{1}\left(e+d_{y}\right)+\left\langle U_{1}^{-1},\left[d_{m_{i}}\right]_{i=1}^{N}\right\rangle .
\end{aligned}
$$

Moreover the critical manifold $\mathcal{M}_{c}=\left\{\tilde{m}_{i}=0, i=2 \ldots, N\right\}$ is exponentially attractive

Proof. Let $\tilde{A}_{1}:=\varepsilon \tilde{A}$. Then $\tilde{A}_{1}$ has spectrum $\varepsilon \lambda_{2}, \ldots, \varepsilon \lambda_{N}$, with $\operatorname{Re}\left(\lambda_{i}\right)<0$ and $\varepsilon \min _{j=2, \ldots, N}\left|\operatorname{Re}\left(\lambda_{j}\right)\right|=1$. The closedloop dynamics (3) then reads

$$
\begin{aligned}
\tau_{g} \dot{g}_{i} & =-g_{i}+[U \tilde{m}]_{i}+d_{g_{i}} \\
\dot{\tilde{m}}_{1} & =\lambda_{1} \tilde{m}_{1}+b_{1}\left(e+d_{y}\right)+\left\langle U_{1 \cdot}^{-1},\left[d_{m_{i}}\right]_{i=1}^{N}\right\rangle \\
\varepsilon\left[\dot{\tilde{m}}_{i}\right]_{i=2}^{N}=\tilde{A}_{1}\left[\tilde{m}_{i}\right]_{i=2}^{N} & +\varepsilon\left(\left[b_{i}\right]_{i=2}^{N}\left(e+d_{y}\right)+\right. \\
& \left.+\left\langle U_{i \cdot}^{-1},\left[d_{m_{j}}\right]_{j=1}^{N}\right\rangle_{i=2}^{N}\right) .
\end{aligned}
$$

Because $\tilde{A}_{1}$ is Hurwitz, in the singular limit $\varepsilon \rightarrow 0$, the model reduces to the slow dynamics (5) defined on the critical manifold $\mathcal{M}_{c}=\left\{\tilde{m}_{i}=0, i=2 \ldots, N\right\}$, which is also exponentially attractive.

We will now show that the reduced dynamics (5) realizes an almost perfect integral controller along the dominant eigenvector associated to the positive molecular regulatory network dynamics defined by $A$. Behavior away from the singular limit is discussed afterward. The following proposition follows by straightforward computations (omitted).

Proposition 3. Let $T_{\lambda_{1}}(s)=\left(\tau_{g} s+1\right)\left(s-\lambda_{1}\right)$ and $\tilde{c}_{1}=$ $\sum_{i=1}^{N} c_{i}\left[v_{1}\right]_{i}$. The transfer output-error functions associated to the reduced model (5) are:

- $G_{r \rightarrow e}(s)=\frac{T_{\lambda_{1}}(s)}{T_{\lambda_{1}}(s)+b_{1} \tilde{c}_{1}}$;

- $G_{d_{g_{i}} \rightarrow e}(s)=\frac{c_{i}\left(s-\lambda_{1}\right)}{T_{\lambda_{1}}(s)+b_{1} \tilde{c}_{1}}$

- $G_{d_{y} \rightarrow e}(s)=\frac{b_{1} \tilde{c}_{1}}{T_{\lambda_{1}}(s)+b_{1} \tilde{c}_{1}}$;

- $G_{d_{m_{i}} \rightarrow e}(s)=\frac{\tilde{c}_{1} U_{1 i}^{-1}}{T_{\lambda_{1}}(s)+b_{1} \tilde{c}_{1}}$.

Observing that $T_{0}(0)=0$, it follows that if $b_{1} \tilde{c}_{1}>0$ and $d_{y}=d_{m_{i}}=0$, then for $\lambda_{1}=0$ the positive regulatory network dynamics achieve perfect regulation of $\left\langle\left[\mathrm{Ca}^{2+}\right]\right\rangle_{T}$, robustly to maximal conductance disturbances, by modulating mRNA expression along the dominant eigenvector of the positive dynamics. This regulation is robust both to output and mRNA disturbances, in the sense that the associated transfer functions have only poles with negative real parts. If $\lambda_{1}<0$, then regulation is almost perfect, with output error of order $\mathcal{O}\left(\lambda_{1}\right)$.

The condition $b_{1} \tilde{c}_{1}>0$ imposes constraints on the dominant and non-dominant eigenvector structure such that the regulation goal is achievable. In particular, if $U$ happens to 
be unitary, then $b_{1}=\sum_{i=1}^{N}\left[v_{1}\right]_{i}>0$ because the dominant eigenvector is positive. The same is true if $U$ is not far from being unitary. Then, the condition $\tilde{c}_{1}=\sum_{i=1}^{N} c_{i}\left[v_{1}\right]_{i}>0$ imposes that the dominant eigenvector components, to which the related mRNA variables converge, are compatible with the effect that the associated ionic conductances have on $\left\langle\left[\mathrm{Ca}^{2+}\right]\right\rangle_{T}$. In particular, eigenvector components associated to variables that increase $\left\langle\left[\mathrm{Ca}^{2+}\right]\right\rangle_{T}$ should be large as compared to components that decrease it.

The following proposition shows that the neuronal homeostatic controller extended with a positive molecular regulatory network robustly regulates the ionic channel conductances in order to maintain stable linear relationships between them, in spite of disturbances and, independently of initial conditions. The proof is omitted for space constraint, but follows from elementary computations.

Proposition 4. Let $\tilde{g}_{j}=\sum_{i=1}^{N} P_{j i}^{-1} g_{i}$.

- $\tilde{g}_{1}=\frac{1}{\tau_{g} s+1}\left(\tilde{m}_{1}+\left\langle P_{1}^{-1},\left[d_{g_{i}}\right]_{i=1}^{N}\right\rangle\right)$;

- $\tilde{g}_{j}=\frac{1}{\tau_{g} s+1}\left\langle P_{j .}^{-1},\left[d_{g_{i}}\right]_{i=1}^{N}\right\rangle, \quad j=2, \ldots, N$;

- $G_{d_{m_{i}} \rightarrow \tilde{m}_{1}}(s)=\frac{\tau_{g} s+1}{T_{\lambda_{1}}(s)+b_{1} \tilde{c}_{1}} P_{1 i}^{-1}$;

- $G_{d_{g_{i}} \rightarrow \tilde{m}_{1}}(s)=\frac{-c_{i}}{T_{\lambda_{1}}(s)+b_{1} \tilde{c}_{1}}$.

Observe that $\tilde{g}_{1}$ is the projection of the maximal conductance vector along the dominant eigenvector $v_{1}$, whereas $\tilde{g}_{j}$, $j=2, \ldots, N$ are the projection in the $N-1$ orthogonal directions to $v_{1}$. Proposition 4 shows that, modulo disturbances and robustly to them, the vector of maximal conductances converges to the subspace generated by the dominant eigenvector $v_{1}$, corresponding to the linear relationships

$$
\frac{g_{i}}{g_{j}}=\frac{\left[v_{1}\right]_{i}}{\left[v_{1}\right]_{j}}
$$

Along this subspace, the vector of maximal conductances behaves as a lagged version of the variable $\tilde{m}_{1}$, which is expected to exhibit large fluctuations in order to achieve (almost) perfect regulation of $\left\langle\left[\mathrm{Ca}^{2+}\right]\right\rangle_{T}$ and compensate for disturbances. How much $\tilde{m}_{1}$ fluctuates in response to disturbances, and thus how much maximal conductances vary along the correlation subspace depends on $\lambda_{1}$. Because both the direction $v_{1}$ and the associated slow eigenvalue $\lambda_{1}$ can be modulated (e.g., during the circadian period), our model provides a simple way to explain how both the linear relationships between maximal conductances and the associated degree of variability can be tuned, while remaining robust to disturbances and insensitive to initial conditions.

Away from the singular limit considered until now, in which the fast eigenvalues are infinitely fast, the unperturbed ( $d_{y}=d_{g_{i}}=d_{m_{i}}=0$ ) behavior follows from standard results in singularly perturbed system [4]. In particular, because the critical manifold is normally hyperbolic (i.e., the fast dynamics is exponentially asymptotically stable - see Theorem 1) for $\varepsilon>0$ and sufficiently small, trajectories of the closed loop dynamics (3) are expected to lie in a $\mathcal{O}(\varepsilon)$-neighborhood of the critical manifold $\left\{\tilde{m}_{j}=0, j=2, \ldots, N\right\}$ and thus maximal conductance to lie in a $\mathcal{O}(\varepsilon)$-neighborhood of the linear correlation subspace $\left\{g_{i} / g_{j}=\left[v_{1}\right]_{i} /\left[v_{1}\right]_{j}\right\}$. The effect of disturbances away from the singular limit, in particular, how far from the critical manifold and the linear correlation subspace trajectories spread, can be studied by computing transfer functions of the fast variables $\tilde{m}_{j}, j=2, \ldots, N$. The proof of the following proposition is elementary (omitted).

Proposition 5. The following holds.

- $G_{d_{m_{i}} \rightarrow \tilde{m}_{j}}(s)=\mathcal{O}(\varepsilon) \bar{G}_{d_{m_{i}} \rightarrow \tilde{m}_{j}}(s), j=2, \ldots, N$,

- $G_{d_{g_{i}} \rightarrow \tilde{m}_{j}}(s)=\mathcal{O}(\varepsilon) \bar{G}_{d_{g_{i}} \rightarrow \tilde{m}_{j}}(s), \quad j=2, \ldots, N$, where $\bar{G}_{d_{m_{i}} \rightarrow \tilde{m}_{j}}(s), \bar{G}_{d_{g_{i}} \rightarrow \tilde{m}_{j}}(s)$ are Hurwitz.

Thus, for $\varepsilon$ sufficiently small, the fast variable are weakly (i.e., $\mathcal{O}(\varepsilon)$ ) sensitive to disturbances. This implies that the correlation set, lying in an $\mathcal{O}(\varepsilon)$ neighborhood of the critical manifold and to which the conductances converge for $d_{y}=$ $d_{g_{i}}=d_{m_{i}}=0$, slightly fattens in the presence of disturbances. In particular, there exists an open set of width $\mathcal{O}(\varepsilon)$ around the unperturbed correlation set that attracts all trajectories. How fat this set is along each of the orthogonal directions to $v_{1}$ depends on the precise non-dominant eigenvalue and eigenvector structure of the molecular regulatory network, a feature that can also be modulated (i.e., during the circadian cycle) to further tune the variability of the model.

Remark 3. Adding leaky terms to the original integral homeostatic controller (1) would solve its robustness issues by avoiding unbounded states but, as opposed to the positive network homeostatic controller, the correlation subspace would not be strongly attractive for this leaky integral controller. Thus, the presence of disturbances would again lead to the disruption of conductance correlation.

\section{Simulations on a DETAILED BiOlOGiCAL MODEL}

To test the performance of the proposed neuronal regulator, we couple equation (3) with the detailed electrophysiological neuron model described in [10]. This model has six voltage and calcium gated currents, eleven state variables, and it can reproduce a variety of electrical behaviors. The maximal conductances of the model are regulated accordingly to (3). Maximal conductances and mRNA variables are initialized at small (close to zero) initial conditions. To simulate the effect of a circadian modulation, the Metzler matrix $A$, determining the homeostatic controller positive network structure and its dominant direction, is supposed to vary periodically on a slower timescale than mRNA and maximal conductance dynamics. In particular, we pick $A(t)=\sin \left(t /\left(2 T_{c}\right)\right)^{2} A_{1}+$ $\left(1-\sin \left(t /\left(2 T_{c}\right)\right)^{2}\right) A_{2}$, where $A_{1}$ and $A_{2}$ are two Metzler matrix and $T_{c}$ is the simulated circadian period. The dominant eigenvectors of the two matrices $A_{1}, A_{2}$ define conductance correlations corresponding to bursting and tonic spiking type, respectively. White noise was added to all mRNAs and maximal conductances dynamics.

Figure 3 shows the outcome of the resulting simulations. Conductances grow exponentially until the $\left\langle\left[\mathrm{Ca}^{2+}\right]\right\rangle_{T} \approx$ $C a_{\text {tgt }}$ and then roughly stabilize. The presence of strong disturbances makes the subsequent time course highly variable but, because of the controller positive network, conductances vary in a correlated fashion. Embedded in the noisy trajectories, the presence of a slow circadian modulation is respon- 


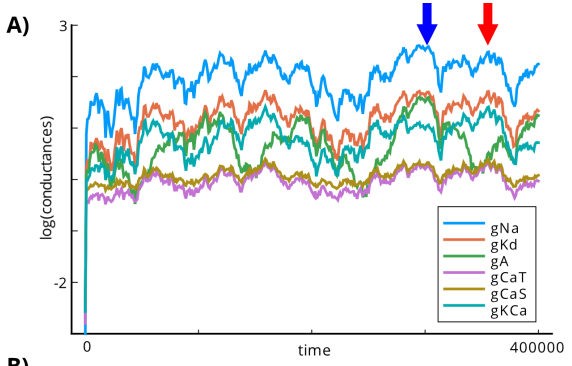

B)
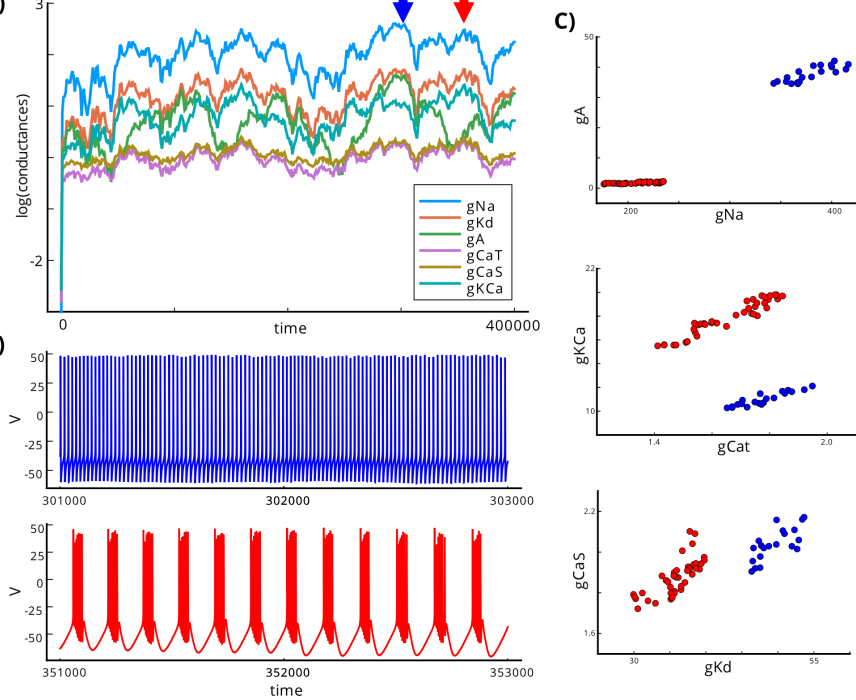

Fig. 3. Output of the proposed positive molecular homeostatic controller A. Simulated maximal conductance time course. The two arrows indicate the locations of the two time windows of length $2000 \mathrm{~ms}$ used to draw the plots in $\mathrm{B}$ and $\mathrm{C}$. Blue arrow corresponds to $A(t) \approx A_{2}$. Red arrow corresponds to $A(t) \approx A_{1}$. B. Time course of the membrane potential during $2 s$ time windows centered at the arrows in A (color-coded). In the top plot the cell is regulated to exhibit tonic spiking activity. In the bottom plot the cell is regulated to exhibit bursting activity. C. Three pairs of conductance correlations during the time windows indicated by the arrows in A. Full code and parameters used in the simulations available upon email request to the first author.

sible for changing the correlation direction, thus modulating the regulated neuronal activity, i.e., the cell is periodically regulated between a tonic spiking type and a bursting type. Correlations are drawn by sampling the conductance evolution in the indicated short time window, which simulates the experimental procedure of measuring different cells' conductances at roughly the same circadian time.

\section{DISCUSSION}

We derived a revised model of neuronal regulation that maintains the biological plausibility and simplicity of the original model proposed in [17] but also captures robustness and tuneability properties of neuronal regulation in a more biologically sound way. The development of controltheoretical models of neuronal homeostasis is also valuable for biologically-grounded control-theoretical approaches to neuronal diseases. The model in [17] already provided insights into how homeostatic compensation can lead to pathological neuronal behaviors. Extending this model with a biologically substantiated molecular regulatory network allows to study from a control-theoretical perspective other types of interactions between neuronal homeostasis and disease, in particular those associated with the disregulation of specific molecular pathways [15], [20].
[2] C. Briat, A Gupta, and M. Khammash. Antithetic integral feedback ensures robust perfect adaptation in noisy biomolecular networks. Cell systems, 2(1):15-26, 2016.

[3] G. Drion, A. Franci, J. Dethier, and R. Sepulchre. Dynamic input conductances shape neuronal spiking. eneuro, 2(1), 2015.

[4] N. Fenichel. Geometric singular perturbation theory for ordinary differential equations. Journal of Differential Equations, 31(1):53-98, 1979.

[5] S. Finkbeiner and M.E. Greenberg. Ca2+ channel-regulated neuronal gene expression. Journal of neurobiology, 37(1):171-189, 1998.

[6] A. Franci, G. Drion, V. Seutin, and R. Sepulchre. A balance equation determines a switch in neuronal excitability. PLoS Comput Biol, 9(5):e1003040, 2013.

[7] G. Giordano and C. Altafini. Interaction sign patterns in biological networks: from qualitative to quantitative criteria. In 2017 IEEE 56th Annual Conference on Decision and Control, pages 5348-5353. IEEE, 2017.

[8] M.S. Goldman, J. Golowasch, E. Marder, and L.F. Abbott. Global structure, robustness, and modulation of neuronal models. The Journal of Neuroscience, 21(14):5229-5238, 2001.

[9] J. Golowasch. Neuromodulation of central pattern generators and its role in the functional recovery of central pattern generator activity. Journal of neurophysiology, 122(1):300-315, 2019.

[10] Z. Liu, J. Golowasch, E. Marder, and L.F. Abbott. A model neuron with activity-dependent conductances regulated by multiple calcium sensors. Journal of Neuroscience, 18(7):2309-2320, 1998.

[11] E. Marder and J-M. Goaillard. Variability, compensation and homeostasis in neuron and network function. Nature Reviews Neuroscience 7(7):563-574, 2006.

[12] E. Marder, T. O'Leary, and S. Shruti. Neuromodulation of circuits with variable parameters: Single neurons and small circuits reveal principles of state-dependent and robust neuromodulation. Annual Review of Neuroscience, 37:329-347, 2014

[13] D.A. McCormick and T. Bal. Sleep and arousal: thalamocortical mechanisms. Annual review of neuroscience, 20(1):185-215, 1997.

[14] J.S. Menet, J. Rodriguez, K.C. Abruzzi, and M. Rosbash. Nascentseq reveals novel features of mouse circadian transcriptional regulation. elife, 1:e0011, 2012.

[15] T. Nagai, W. Shan, and K. Yamada. Exploring molecular targets for epilepsy treatment from the perspective of neuronal homeostasis. Journal of the Pharmaceutical Society of Japan, 139(6):923-929, 2019.

[16] D. Noutsos. On perron-frobenius property of matrices having some negative entries. Linear Algebra and its Applications, 412(2-3):132153,2006

[17] T. O'Leary, A.H. Williams, A. Franci, and E. Marder. Cell types, network homeostasis, and pathological compensation from a biologically plausible ion channel expression model. Neuron, 82(4):809-821, 2014.

[18] T. O'Leary and D.J.A. Wyllie. Neuronal homeostasis: time for a change? The Journal of physiology, 589(20):4811-4826, 2011.

[19] D.J. Schulz, J-M. Goaillard, and E. Marder. Quantitative expression profiling of identified neurons reveals cell-specific constraints on highly variable levels of gene expression. Proceedings of the National Academy of Sciences, 104(32):13187-13191, 2007.

[20] B. Styr, N. Gonen, D. Zarhin, A. Ruggiero, R. Atsmon, N. Gazit, G. Braun, S. Frere, I. Vertkin, I. Shapira, and I. Slutsky. Mitochondrial regulation of the hippocampal firing rate set point and seizure susceptibility. Neuron, 102(5):1009-1024, 2019.

[21] T. Tran, C.T. Unal, D. Severin, L. Zaborszky, H.G. Rotstein, A. Kirkwood, and J. Golowasch. Ionic current correlations are ubiquitous across phyla. Scientific reports, 9(1):1-9, 2019.

[22] C.T. Unal, J. Golowasch, and L. Zaborszky. Adult mouse basal forebrain harbors two distinct cholinergic populations defined by their electrophysiology. Frontiers in behavioral neuroscience, 6:21, 2012.

\section{REFERENCES}

[1] D. Angeli and E.D. Sontag. Monotone control systems. IEEE Transactions on Automatic Control, 48(10):1684-1698, 2003. 